\title{
How to monitor systemic lupus erythematosus in daily practice
}

\author{
Ioana Saulescu, Ruxandra Ionescu \\ Department of Internal Medicine and Rheumatology, "Sfanta Maria" Clinical Hospital, Bucharest, Romania \\ "Carol Davila" University of Medicine and Pharmacy, Bucharest, Romania
}

\begin{abstract}
Systemic lupus erythematosus (SLE) is one of the most heterogeneous autoimmune disease that can virtually affect any organ, characterized by different course of evolution and being associated with increased morbidity due to activity, that can also contribute to chronic damage. It is imperative to have a good monitoring schedule according to different situations in order to take the best decision.
\end{abstract}

Keywords: disease activity, systemic lupus erythematosus, activity index

\section{INTRODUCTION}

Systemic lupus erythematosus (SLE) is one of the most heterogeneous autoimmune disease that can virtually affect any organ. Not only that clinical manifestations are diverse, but the course of the disease can vary from longstanding quiescent phenotype to chronic remitting and relapsing disease, with relapses being associated with increased morbidity due to disease activity, but also by contribution to chronic damage (1). Therefore, physicians should adapt treatment according to disease activity, flares or irreversible damage accrual. From this perspective, it is imperative to have a good monitoring schedule, adapted to disease progression. At this moment, for SLE patients there are available guidelines for treatment and evaluation that allow to take proper therapeutic decisions (2-4). In Romania, belimumab, a human monoclonal antibody that inhibits B-cell activating factor, is reimbursed since 2018 and this treatment is prescribed after a thorough evaluation according to an approved protocol with clear criteria for initiation and maintenance of treatment (5). In this situation, the Romanian Register for Rheumatic Diseases (RRBR) offers an useful tool to monitor patients and to verify the imposed criteria.

Because SLE is a multi-organ disease, evaluation of patients should be done in order to recognize types of organ involved. Severity of the disease should be quantified by specific scores and should be mandatory to differentiate between organ threating involvement versus non-threatening involvement. Another important issue is to differentiate between activity and damage.

The variety of organ involvement imposes a thorough evaluation at each visit in order to avoid overtreating or undertreating and to limit the undesired consequences of both. From this perspective, it is not easy all the time to differentiate between signs and symptoms related to active disease or chronic damage, damage that might be related to the disease itself or even to treatment, with major impact on treatment decisions. Fortunately, at this moment there is a validated and reliable tool in order to do just that $(6,7)$. One of the most challenging diseases, SLE has, in most of the cases, a fluctuating course over time. Therefore, it is very important to use this measuring tool in everyday practice (7).

\section{INDICES FOR ACTIVITY AND DAMAGE}

At this moment, there are validated indices both for activity and damage. Since the disease is so heterogeneous, validation of these scores implied a lot of effort, at least for the activity score. Ideally, they should capture, if possible, evolution of organ in- 
volvement severity from one visit to another, but also the flares. Correct assessment of disease activity in daily practice is mandatory for proper medical decision and should be based on practical scores. Some of them are global indices: SLEDAI (Systemic Lupus Erythematosus Disease Activity Index), SLAM (Systemic Lupus Activity Measure), but others quantify intention to treat, for example BILAG (British Isles Lupus Assessment Group) $(1,8,9)$. Some are more prone to be used in clinical settings, such as SLEDAI, Physician Global Assessment (PGA) or SELENA-SLEDAI Flare Index, while others are more suitable for clinical trials, such as BILAG. This is why RRBR uses the first three, aiming to be a friendly tool for rheumatologists.

The SLEDAI index is probably the most used worldwide. It takes less than 10 minutes to be completed and it is very simple to use. It has simple definitions and it is formed from 16 clinical variables and 8 laboratory variables. The weight of these variables is different according to the prognostic impact. The sum of the items gives the result and categorizes the patient in remission, mild, moderate or severe disease. An important flaw of the SLEDAI is the fact that it does not capture evolution of the same organ system (10).

Physician Global Assessment (PhGA) is a visual analogue scale that estimate activity on a scale from 0 to 3 . It should capture the evolution of the disease at every evaluation.

SELENA-SLEDAI Flare Index classifies severe versus mild or moderate flares and has the advantage that it captures the therapeutic attitude.

How we define mild, moderate and severe lupus activity?

Mild disease activity means that the disease is stable with no life-threating organ involvement. In this situation damage is not expected to occur, the SLEDAI score is less than 6 and PhGA is less than 1.

Moderate disease means a more serious organ involvement that, if left untreated, could cause organ damage. In this situation, SLEDAI is between 6 and 12 and PhGA is between 1 and 2.5.

Severe disease means that organ involvement is life-threatening (central nervous system, renal, cardiac or pulmonary involvement) and requires aggressive immunosuppression. Damage will occur even in the presence of appropriate treatment. In this situation, SLEDAI is above 12 and PhGA is grater then $2.5(3,10,11)$.

Currently, because of the prolonged use of hydroxychloroquine associated with a better under- standing of SLE pathogenesis and a more guided treatment (4), a larger proportion of patients have a stabile mild or low disease activity. Damage accrual is a continuous process, related to immune and inflammatory state or secondary to treatment (glucocorticoids, immunosuppressants) or comorbidities. Since 1996, a damage index elaborated by Systemic Lupus International Collaborating Clinics has been widely used (the SLICC/ACR Damage Index). High index scores early during the disease course is associated with poor prognosis and increased mortality (12).

What will be the purpose of using these different scores in daily practice? They allow physicians to have a clear image of disease activity, flares and organ damage, giving the opportunity to take the best treatment decisions. Moreover, these tools help to establish the frequency of evaluations: at least every 1-3 months for active disease or every 6-12 months for patients with stable or low disease activity (3).

Beside disease evaluation, lifestyle guidance is mandatory for increasing the quality of life. Photoprotection should be recommended for all patients with or without skin involvement. Smoking cessation is advised in order to lower cardiovascular risk (which is increased in these patients by the disease itself), physical exercise should be recommended to patients on glucocorticoids in order to prevent weight gain and bone loss.

\section{EVALUATION OF COMORBIDITIES}

An important issue of each clinical appointment of SLE patients is related to correct evaluation of comorbidities. Some of the most important are cardiovascular comorbidity and its risk factors, pro-thrombotic state related to secondary antiphospholipid syndrome or osteoporosis.

A similar attention should be focused on family planning. It is well documented that SLE has a female predominance during the reproductive years. Family planning and contraception solutions must be discussed from the moment of diagnosis and on regular basis onwards. The last decades came with a tremendous change regarding this topic. Maternal and fetal outcomes improved and now pregnancy is possible, preferably with a correct timing assessed by rheumatologists. For this purpose, activity of the disease and life-threatening organ involvement, such as renal involvement, must be assessed prior to conception. A pregnancy should be planned after at least 
6 months without flares. Treatment should be adjusted in order to avoid exposure to prohibited medication, immunology must be re-checked especially for secondary antiphospholipid antibody and for the presence of anti-Ro antibodies. The relation between congenital heart block and anti-Ro antibodies must be discussed with the future SLE mother. A multidisciplinary approach including a rheumatologist and a gynecologist will increase the likelihood for a good prognosis, avoiding stopping essential permitted therapies such as hydroxychloroquine or azathioprine (13).

Infections are very frequent in SLE patients because of immune abnormalities or because of immunosuppressive treatments. Prompt recognition and treatment of infections might prevent flares. Season- al influenza and pneumococcal vaccination are strongly recommended during stable disease. Live vaccines must be avoided, especially in patient under immunosuppression (14).

Osteopenia and osteoporosis are very frequent in SLE patients. Bone mineral density must be assessed at diagnosis of SLE and then annually, especially if the patients are on glucocorticoids (4).

\section{CONCLUSION}

When treating such a complex disease, with a broad type of organ involvement and different types of disease course, it is better to follow a routine for every case. This will allow to take the best treatment decisions and to avoid complications and damage accrual.

Conflict of interest: none declared Financial support: none declared

\section{REFERENCES}

1. Fernando MMA, Isenberg DA. How to monitor SLE in routine clinical practice. Ann Rheum Dis. 2005; 64: 524-527.

2. Mosca M, Tani $C$, Aringer $M$ et al. European League Against Rheumatism Recommendations for monitoring patients with Systemic Lupus Erythematosus in Clinical Practice and in observational studies. Ann Rheum Dis. 2010; 69(7):1269-1274.

3. Gordon C, Amissah-Arthur MB, Gayed M. The British Society for Rheumatology guideline for the management of systemic lupus erythematosus in adults. Rheumatology, 2018; 57 (1):e1-e45.

4. Fanouriakis A, Kostopoulou M, Alunno A et al. 2019 update of the EULAR recommendations for the management of systemic lupus erythematosus. Ann Rheum Dis. 2019; 78:736-745.

5. Monitorul Oficial al României nr. 754 bis / 31 viii 2018; 195-198.

6. Mosca M, Tani C, Aringer M et al. Development of quality indicators to evaluate the monitoring of SLE patients in routine clinical practice. Autoimmun Rev. 2011; 10 (7):383-388.

7. Ruperto N, Hanrahan LM, Alarcon GS et al. International consensus for a definition of disease flare in lupus. Lupus, 2011; 20:453-462.

8. Stand V, Gladman D, Isenberg D et al. Outcomes measure to be used in clinical trials in SLE. J Rheumatol. 1999; 26:490-498.

9. Haq I, Isenberg DA. How does one assess and monitorpatients with systemic lupus erythematosus in daily clinical practice. Best Pract Res Clin Rheumatol. 2002; 16:181-194.
10. Gladman DD, Ibanez D, Urowitz MD. Systemic Lupus Erythematosus Disease Activity Index 2000. J Rheumatol. 2002; 29 (2):288-291.

11. Aranow $C$. A pilot study to determine the optimal timing of the Physician Global Assessment (PGA) in patients with systemic lupus erythematosus. Immunol Res. 2015 Dec;63(1-3):167-9.

12. Rahman $P$, Gladman DD, Urowitz MB, Hallett D, Tam LS. Early damage as measured by the SLICC/ACR damage index is a predictor of mortality in systemic lupus erythematosus. Lupus. 2001; 10(2):93-6.

13. Andreoli L, Bertsias GK, Agmon-Levin N et al. EULAR recommendations for womens health and the management of family planning, assisted reproduction, pregnancy and menopause in patients with systemic lupus erythematosus and/ or antiphospholipidic syndrome. Ann Rheum Dis. 2017; 75 (3): 476-485.

14. Furer V, Rondaan C, Heijstek MW et al. 2019 update of EULAR recommendations for vaccination in adult patients with autoimmune inflammatory rheumatic diseases. Ann Rheum Dis. 2020 Jan; 79(1):39-52 